\title{
THE GAS TEMPERATURE OF STARLESS CORES IN PERSEUS
}

\author{
S. Schnee ${ }^{1}$, E. Rosolowsky ${ }^{2,3}$, J. Foster ${ }^{2}$, M. Enoch ${ }^{4}$, And A. Sargent ${ }^{1}$ \\ ${ }^{1}$ Division of Physics, Mathematics and Astronomy, California Institute of Technology, 770 South Wilson Avenue, Pasadena, CA 91125, USA; \\ schnee@astro.caltech.edu \\ ${ }^{2}$ Harvard-Smithsonian Center for Astrophysics, 60 Garden Street, Cambridge, MA 02138, USA \\ ${ }^{3}$ University of British Columbia Okanagan, 3333 University Way, Kelowna, BC V1V 1V7, Canada \\ 4 Department of Astronomy, University of California, Berkeley, CA 94720, USA \\ Received 2008 July 25; accepted 2008 September 29; published 2009 February 9
}

\begin{abstract}
In this paper, we study the determinants of starless core temperatures in the Perseus molecular cloud. We use $\mathrm{NH}_{3}(1,1)$ and $(2,2)$ observations to derive core temperatures $\left(T_{\text {kin }}\right)$ and data from the COMPLETE Survey of Star-Forming Regions and the c2d Spitzer Legacy Survey for observations of the other core and molecular cloud properties. The kinetic temperature distribution probed by $\mathrm{NH}_{3}$ is in the fairly narrow range of $\sim 9-15 \mathrm{~K}$. We find that cores within the clusters IC348 and NGC1333 are significantly warmer than "field" starless cores, and $T_{\text {kin }}$ is higher within regions of larger extinction-derived column density. Starless cores in the field are warmer when they are closer to Class 0/I protostars, but this effect is not seen for those cores in clusters. For field starless cores, $T_{\text {kin }}$ is higher in regions in which the ${ }^{13} \mathrm{CO}$ line width and the $1.1 \mathrm{~mm}$ flux from the core are larger, and $T_{\mathrm{kin}}$ is lower when the the peak column density within the core and average volume density of the core are larger. There is no correlation between $T_{\text {kin }}$ and ${ }^{13} \mathrm{CO}$ line width, $1.1 \mathrm{~mm}$ flux, density, or peak column density for those cores in clusters. The temperature of the cloud material along the line of sight to the core, as measured by CO or far-infrared emission from dust, is positively correlated with core temperature when considering the collection of cores in the field and in clusters, but this effect is not apparent when the two subsamples of cores are considered separately.
\end{abstract}

Key words: stars: formation - ISM: molecules

Online-only material: color figure

\section{INTRODUCTION}

Starless cores are the link between molecular clouds and Class 0 protostars. They are smaller and denser $(r \sim 0.1 \mathrm{pc}, n \gtrsim$ $10^{4} \mathrm{~cm}^{-3}$ ) than, and kinematically distinct from, the molecular clouds in which they are found (Goodman et al. 1998). Because starless cores have no source of internal heating, the temperature of a starless core is determined by external illumination and self-shielding. The temperatures of starless cores are set by the interstellar radiation field (ISRF), and in some cases luminous nearby stars, attenuated by the molecular cloud. The temperature profile within a starless core is determined by the heating of its outer layers and the internal extinction, making the centers of starless cores colder than their outer layers (Evans et al. 2001; Stamatellos et al. 2007; Zucconi et al. 2001).

The temperatures of starless cores can be inferred from thermal emission from dust or from molecular lines. Deriving core temperatures from continuum emission is complicated by uncertainties in the dust emission spectrum. For instance, in cold and dense regions, dust grains are expected to coagulate and become covered in icy mantles, creating variation in the emissivity spectral index (Ossenkopf \& Henning 1994). In addition, below a density of $\sim 3 \times 10^{4}$, the dust temperature is not coupled to the gas temperature (Goldsmith 2001; Galli et al. 2002), which is the more important quantity because the gas makes up $99 \%$ of the mass. One of the best tracers of gas temperature is the rotation-inversion lines of $\mathrm{NH}_{3}$. Ammonia is particularly well suited for this type of study because (1) $\mathrm{NH}_{3}$ does not seem to deplete onto dust grains in starless cores, and in fact may have an enhanced abundance where $\mathrm{CO}$ is depleted (Tafalla et al. 2002), (2) $\mathrm{NH}_{3}$ has hyperfine splitting of its inversion lines, making fits to the temperature, velocity, and optical depth more certain, and (3) the $\mathrm{NH}_{3}(1,1)$ and $(2,2)$ transitions are separated by a small difference in frequency, so it is possible to simultaneously observe both sets of lines. In this paper, we use $\mathrm{NH}_{3}(1,1)$ and $(2,2)$ observations (Rosolowsky et al. 2008) to derive the temperature of starless cores. We examine whether these data support the several models of the relationship between core properties and the molecular cloud where they are found (e.g., Evans et al. 2001; Bethell et al. 2004; Stamatellos et al. 2004).

Perseus is one of the Gould's belt molecular clouds included in the Spitzer Legacy project "From Molecular Cores to PlanetForming Disks" (c2d1; Evans et al. 2003) and the COMPLETE Survey of Star-Forming Regions (Ridge et al. 2006), at a distance of 250 pc (Černis \& Straižys 2003; Belikov et al. 2002). Because it is so well observed, there is a wealth of data on the cores and the cloud that enables us to study the influences of various internal and environmental factors on the temperature of 50 starless cores in Perseus.

\section{DATA}

\subsection{Far-Infrared}

As described in Schnee et al. (2008), we used far-infrared (FIR) emission maps at 70, 100, and $160 \mu \mathrm{m}$ to derive the dust temperature and column density of the Perseus molecular cloud. The 70 and $160 \mu \mathrm{m}$ maps were obtained as part of the $\mathrm{c} 2 \mathrm{~d}$ survey (Rebull et al. 2007), and the $100 \mu \mathrm{m}$ map was taken from the infrared interferometer spectrometer (IRIS) reprocessing of the IRAS all-sky maps (Miville-Deschênes \& Lagache 2005). The resolution of the emission-derived column density $\left(N_{\mathrm{H}}\right)$ and dust temperature $\left(T_{\mathrm{d}}\right)$ maps with which we will compare the $\mathrm{NH}_{3}$ temperature $\left(T_{\text {kin }}\right)$ is $40^{\prime \prime}$, as set by the $160 \mu \mathrm{m}$ data. 
The dust temperature is calculated from the ratio of the observed flux at 70 and $160 \mu \mathrm{m}$, taking into account the color correction at both wavelengths and the emission from transiently heated very small dust grains at $70 \mu \mathrm{m}$. The column density is derived from the flux at $160 \mu \mathrm{m}$ and the dust temperature, allowing for a variable dust emissivity and normalized to the extinction measured from near-infrared (NIR) reddening. Maps of the emission-derived dust temperature and column density, and the procedure used to make the maps, are presented in detail in Schnee et al. (2008).

\subsection{Near-Infrared}

Data from the final release of the Two Micron All Sky Survey (2MASS) point-source catalog was used to create a large-scale extinction map of Perseus as part of the COMPLETE Survey (Ridge et al. 2006). The NICER algorithm estimates the column density toward background stars by comparing their observed colors with the (assumed) intrinsic colors (Lombardi \& Alves 2001). Although we expect the extinction map to be our leastbiased estimate of the cloud column density (Schnee et al. 2006; Goodman et al. 2008), its 5' resolution is roughly 10 times coarser than our other maps, which smooths out density gradients and makes it somewhat harder to compare the cloud and core properties.

\section{3. $\mathrm{NH}_{3}(1,1)$ and $(2,2)$}

We used observations of 193 dense cores and core candidates in the Perseus molecular cloud using the $100 \mathrm{~m}$ Robert F. Byrd Green Bank Telescope (GBT) as described in Rosolowsky et al. (2008). For each target, single-pointing (31" FWHM) observations were made of the $\mathrm{NH}_{3}(1,1)$ and $(2,2)$ lines with $0.024 \mathrm{~km} \mathrm{~s}^{-1}$ spectral resolution. The targets for the ammonia survey were drawn, in order of precedence, from (1) the locations of millimeter cores in the Bolocam survey of the region (Enoch et al. 2006), (2) the locations of submillimeter cores in the Submillimeter Common-User Bolometric Array (SCUBA) survey of the region (Kirk et al. 2006), (3) sources in the literature survey of Jijina et al. (1999), (4) cold, highcolumn-density objects in the dust map produced by Schnee et al. (2008), and (5) sources identified by eye that appear in both the Bolocam and SCUBA maps but were not included in the published catalogs. The starless cores discussed in this paper were drawn from only the first two categories.

The kinetic temperatures of the starless cores (Section 3 describes how we identify which cores are starless) are determined by a simultaneous fit to the $(1,1)$ and $(2,2)$ emission profiles, accounting for collisional deexcitation of the two states. The same fit also yields the width of the $\mathrm{NH}_{3}$ line, which is decomposed into its thermal and nonthermal components based on the temperature determination. Although there are likely gas temperature and density gradients within each starless core on both theoretical and observational grounds (Crapsi et al. 2007), the fitting of the ammonia-derived temperature is essentially an average over the core properties. However, a single-temperature model with uniform excitation conditions is a reasonably good fit to nearly all of the starless cores in this sample. The $\mathrm{NH}_{3}$ observations can be used to accurately derive the kinetic temperatures of starless cores with $8 \mathrm{~K} \leqslant T_{\text {kin }} \leqslant 40 \mathrm{~K}$, where the lower and upper limits are set by sensitivity and transition thermalization, respectively (Walmsley \& Ungerechts 1983). Further details about the observations and data reduction are described in Rosolowsky et al. (2008).

$$
\text { 2.4. }{ }^{12,13} \mathrm{CO}(1-0)
$$

Observations of the ${ }^{12} \mathrm{CO}(1-0)$ and ${ }^{13} \mathrm{CO}(1-0)$ transitions at 115 and $110 \mathrm{GHz}$ were carried at the $14 \mathrm{~m}$ Five College Radio Astronomy Observatory (FCRAO) telescope as part of the COMPLETE Survey. The data have $0.07 \mathrm{~km} \mathrm{~s}^{-1}$ spectral resolution and $40^{\prime \prime}$ spatial resolution. The data reduction and mapping techniques are described in Ridge et al. (2006). The $\mathrm{CO}$ maps cover approximately the same area in Perseus as the c2d survey, giving us a good estimate of the kinematics of the ambient, less dense material in the molecular cloud. Pineda et al. (2008) use this data set to determine the gas temperature and column density in regions in which the ${ }^{13} \mathrm{CO}(1-0)$ transition is optically thin (the ${ }^{12} \mathrm{CO}$ line is assumed to be optically thick everywhere) and the gas is not subthermally excited.

\subsection{1 mm Continuum Maps}

As part of a survey that is complementary to c2d, Enoch et al. (2006) mapped $7.5 \mathrm{deg}^{2}$ of the Perseus molecular cloud at 1.1 $\mathrm{mm}$ with Bolocam at the Caltech Submillimeter Observatory. The resolution is $31^{\prime \prime}$, which matches very well with our $\mathrm{NH}_{3}$ observations, and all $1221.1 \mathrm{~mm}$ detected cores were observed with the GBT. The Bolocam observations are most sensitive to dense cores $\left(n \gtrsim 2 \times 10^{4} \mathrm{~cm}^{-3}\right.$ ) with density contrasts at least 30 times greater than the background cloud, which matches the description of a starless core embedded in a molecular cloud quite well (Bergin \& Tafalla 2007). In addition to using the $1.1 \mathrm{~mm}$ data for target selection, we use the $1.1 \mathrm{~mm}$ derived fluxes and sizes to compare the $T_{\text {kin }}$ with core properties such as mass, column density, and volume density.

\section{IDENTIFICATION OF STARLESS CORES}

To ensure that we study only the starless core population in Perseus, we compare the positions of our $\mathrm{NH}_{3}$ observations with the coordinates of known protostars, as found through the analysis of Enoch et al. (2008a), Jørgensen et al. (2007), and Hatchell et al. (2007). Jørgensen et al. (2007) identify 49 deeply embedded young stellar objects (YSOs) in Perseus using Spitzer/IRAC color cuts, Spitzer/MIPS and 850 $\mu \mathrm{m}$ SCUBA correlations, and SCUBA morphologies. Hatchell et al. (2007) uses NIR/MIR (2MASS/IRAC) detections and/or detections of protostellar outflows to separate starless cores from protostars, and find 56 protostars. Enoch et al. (2008a) identifies 55 protostellar cores out of the total $1221.1 \mathrm{~mm}$ cores in Perseus using the spatial coincidence between $1.1 \mathrm{~mm}$ continuum cores and sources identified in the c2d Delivery Document (Evans et al. 2007; Harvey et al. 2007) as "YSOc" (YSO candidate), "red" or that are $70 \mu \mathrm{m}$ point sources and are not classified as "Galc" (Galaxy candidate). In this paper, we consider only those 50 ammonia pointings that are called "starless" by the various definitions of all three papers, or that are more than 30 " from any identified protostar in any of the three papers. None of the cores we classify as "starless" is identified as a low-luminosity embedded protostar by Dunham et al. (2008). We also require that the starless cores have solid ammonia-derived temperatures and line widths, meaning that $T_{\text {kin }}$ is greater than $2.73 \mathrm{~K}$, the $\mathrm{NH}_{3}$ $(2,2)$ detection is $>5 \sigma$, and the model and observed spectra fit well by eye. Of the 193 observations in the $\mathrm{NH}_{3}$ survey, 137 of the pointings are not coincident with an identified protostar, and 50 of these satisfy the signal-to-noise and goodness-of-fit requirements. 


\section{ANALYSIS}

Here we compare the ammonia-derived temperature with several properties of the starless cores and their environment. We considered only those 50 starless cores with well defined $T_{\text {kin }}$ and line width, so we are able to derive their nonthermal line widths. All were detected in the $1.1 \mathrm{~mm}$ survey of Enoch et al. (2006), so for each we have the total flux, peak flux (from which we derive the peak column density), mass (from the total flux and the $\mathrm{NH}_{3}$ temperature), and mean particle density. The FIRderived dust temperature and column density are considered to be believable as long as they fall within the range $12 \leqslant T_{\mathrm{d}} \leqslant 20$ and $0 \leqslant A_{V} \leqslant 24.5$, which is true except in those regions where the $160 \mu \mathrm{m}$ map is saturated or corrupted by a nearby bright source. There are 46 starless cores with believable FIRderived $T_{\mathrm{d}}$ and $A_{V}$, and all 50 starless cores fall within the area of our NIR-derived extinction map. The Gaussian-fitting algorithm failed to converge when calculating the ${ }^{13} \mathrm{CO}$ line width of $T_{\mathrm{CO}}$ for six cores. The internal properties of our sample of starless cores are listed in Table 1, and the properties of Perseus cloud along the line of sight to the cores are given in Table 2. Although the distance from each starless core to the nearest protostar found in the surveys of Enoch et al. (2008a); Jørgensen et al. (2007), and Hatchell et al. (2007) is known, in our discussion of possible correlations we consider only those with separations of $0.25 \mathrm{pc}$ or smaller.

\section{1. $T_{\text {kin }}$}

The kinetic temperature distribution probed by $\mathrm{NH}_{3}$ is quite narrow, from $\sim 9-15 \mathrm{~K}$, as seen in Figure 1. This may be due to the relatively low angular resolution of the GBT pointings, whose $31^{\prime \prime}$ beam corresponds to probing the inner $0.019 \mathrm{pc}$ $\left(3.9 \times 10^{3} \mathrm{AU}\right)$ of each core. Models of externally heated prestellar cores show that they are only significantly colder than $9 \mathrm{~K}$ within the inner $\sim 10^{3} \mathrm{AU}$ or warmer than $14 \mathrm{~K}$ outside a radius of $\sim 10^{4} \mathrm{AU}$, with variation depending on the strength of the ISRF incident upon the core (Evans et al. 2001; Stamatellos et al. 2004). We are unlikely to be biased by chemical effects, given that $\mathrm{NH}_{3}$ is not seen to deplete in starless cores (e.g., Tafalla et al. 2002). The critical density of $\mathrm{NH}_{3}, \sim 10^{4} \mathrm{~cm}^{-3}$ (Swade 1989), is low compared with the typical gas densities observed in the cores in our sample $\left(10^{4} \mathrm{~cm}^{-3} \leqslant n \leqslant 10^{6} \mathrm{~cm}^{-3}\right)$, so we expect the $\mathrm{NH}_{3}$ observations to sample the full volume of our cores.

The range of temperatures seen in the Perseus starless cores is typical of those seen in other molecular clouds. For instance, Kirk et al. (2007) derived dust temperatures of prestellar cores in Taurus and Ophiuchus and found that most cores fall within the 9-13 K range. The peak of the $T_{\text {kin }}$ distribution shown in Figure 1 is between $10-11 \mathrm{~K}$, which agrees well with the median temperature $(11 \mathrm{~K})$ derived from $\mathrm{NH}_{3}$ observations of the 36 starless cores as observed and identified by Jijina et al. (1999) across many nearby star-forming regions. The lower and upper quartiles of the temperatures of the Jijina et al. (1999) starless core sample $(9.7 \mathrm{~K}$ and $14.0 \mathrm{~K})$ are also a close match to that shown in Figure 1.

Numerical simulations of starless cores have been used to quantify how their temperature profile is affected by variations in many parameters, such as the strength of the ISRF, the density profile, and the amount of extinction provided by the molecular cloud in which the cores are embedded (e.g., Evans et al. 2001; Galli et al. 2002; Stamatellos \& Whitworth 2003; Stamatellos et al. 2004). Evans et al. (2001) calculate that for Bonnor-Ebert spheres with central densities varying by about a factor of 100 (from $10^{5}$ to $10^{7} \mathrm{~cm}^{-3}$ ), the central temperature varies by a factor of $\sim 2(6.5-10 \mathrm{~K})$, but the temperature at a radius of $10^{3.5} \mathrm{AU}$ (the radius of the GBT beam at the distance of Perseus) only varies by $\sim 1 \mathrm{~K}(9-10 \mathrm{~K})$. A doubling of the strength of the ISRF results in a $1 \mathrm{~K}$ increase of the core temperature file over the range of radii probed by the $\mathrm{NH}_{3}$ observations in this study (Evans et al. 2001), while a factor of 100 increase in the ISRF would result in an increase in the core temperature by a factor of $\sim 2$ (Galli et al. 2002). Variations in the dust opacity also affect the temperature of starless core, but Gonçalves et al. (2004) show that this effect is quite small $(<1 \mathrm{~K})$ for the range of dust properties that one expects in these cores. Given that the temperature of a prestellar core (at a radius of $10^{3.5} \mathrm{AU}$ ) is not especially sensitive to its central density, the dust opacity, the strength of the ISRF or the degree of extinction for the range of values that we expect in Perseus, the narrow width of the temperature distribution shown in Figure 1 and its peak at $10 \mathrm{~K}$ are roughly what one would expect.

\section{2. $T_{\text {kin }}$ versus Location}

The presence of local star formation appears to affect $T_{\text {kin }}$. Figure 1 shows the temperature distribution of all the starless cores in Perseus (filled gray histogram), as well as that for starless cores in the clusters NGC1333 and IC348 (open solid histogram) and field starless cores (open dashed histogram). The boundaries for NGC1333 and IC348 are taken from Jørgensen et al. (2007). It is clear that those cores in clusters are warmer than those outside clusters. The probability that the temperature distributions of starless cores inside and outside the clusters have the same mean is $\simeq 10^{-5}$, as calculated by a Wilcoxon ranksum test, the student's $t$-statistic, or the two-sided KolmogorovSmirnov test.

There are reasons to expect that starless cores in clusters would be warmer than those outside clusters. For instance, a cluster of protostars may directly heat their environment. In addition, protostars inject turbulence into the surrounding medium, making it more porous and less effective as a shield from the ISRF. In addition, Jijina et al. (1999) find that dense cores that are not associated with IRAS sources are warmer when found inside the clusters than outside.

Stamatellos et al. (2007) predict that the presence of a nearby Class 0 protostar should not affect the temperature of a nearby starless core at all, and that a nearby Class I protostar will only heat the outer layers of a core, and only by $\sim 1-2 \mathrm{~K}$. We test this by comparing the ammonia-derived temperatures of starless cores in Perseus to the distance to the nearest Class 0 and I protostars (as determined by Enoch et al. 2008b). As shown in Figure 2 and Table 3, we confirm that there is no significant correlation between $T_{\text {kin }}$ and the distance to the nearest protostar for those starless cores in the clusters NGC1333 and IC348. However, there is a significant negative correlation between $T_{\text {kin }}$ and the distance to the nearest Class 0 and/or I protostar for those starless cores in the field (i.e., starless cores further from protostars are colder). It is not at all certain that this result contradicts the prediction of Stamatellos et al. (2007), because we cannot say that nearby protostars heat starless cores, just that warmer cores are found near protostars.

\section{3. $T_{\text {kin }}$ versus Cloud Temperature}

Here we compare the temperatures of starless cores with the temperature of the material in which it is embedded. We 
Table 1

Internal Properties of Starless Cores

\begin{tabular}{|c|c|c|c|c|c|c|c|c|}
\hline $\begin{array}{l}\text { R.A. }{ }^{a} \\
\text { J2000 } \\
\end{array}$ & $\begin{array}{l}\text { Decl. }^{\mathrm{b}} \\
\text { J2000 }\end{array}$ & $\begin{array}{c}T_{\text {kin }}{ }^{\mathrm{c}} \\
(\mathrm{K})\end{array}$ & $\begin{array}{c}\sigma_{\mathrm{NT}}{ }^{\mathrm{d}} \\
\left(\mathrm{km} \mathrm{s}^{-1}\right)\end{array}$ & $\begin{array}{c}1.1 \mathrm{~mm} \mathrm{Flux} \mathrm{e}^{\mathrm{e}} \\
(\mathrm{Jy})\end{array}$ & $\begin{array}{c}\text { Peak } A_{V}{ }^{\mathrm{f}} \\
(\mathrm{mag})\end{array}$ & $\begin{array}{c}\text { Mass }^{\mathrm{g}} \\
\left(M_{\text {solar }}\right)\end{array}$ & $\begin{array}{c}\text { Density }^{\mathrm{h}} \\
\left(\mathrm{cm}^{-3}\right)\end{array}$ & Number $^{\mathrm{i}}$ \\
\hline $3: 25: 07.8$ & $30: 24: 22$ & 9.2 & 0.09 & 0.1 & 9.1 & 0.3 & $1.4 \mathrm{E}+05$ & 1 \\
\hline $3: 25: 09.7$ & $30: 23: 53$ & 9.2 & 0.14 & 0.1 & 10.1 & 0.4 & $3.3 \mathrm{E}+05$ & 2 \\
\hline $3: 25: 10.1$ & $30: 44: 41$ & 10.0 & 0.12 & 0.4 & 9.0 & 0.9 & $2.5 \mathrm{E}+04$ & 3 \\
\hline $3: 25: 17.1$ & $30: 18: 53$ & 10.3 & 0.11 & 1.2 & 10.1 & 2.8 & $4.5 \mathrm{E}+04$ & 4 \\
\hline $3: 25: 26.9$ & $30: 21: 53$ & 9.1 & 0.10 & 1.2 & 12.3 & 3.4 & $5.3 \mathrm{E}+04$ & 6 \\
\hline $3: 25: 46.1$ & $30: 44: 11$ & 11.2 & 0.18 & 0.9 & 14.2 & 1.8 & $8.5 \mathrm{E}+04$ & 11 \\
\hline $3: 25: 48.8$ & $30: 42: 24$ & 9.1 & 0.13 & 0.5 & 34.6 & 1.3 & $3.8 \mathrm{E}+05$ & 13 \\
\hline $3: 25: 50.6$ & $30: 42: 02$ & 9.0 & 0.14 & 0.4 & 29.6 & 1.2 & $3.2 \mathrm{E}+05$ & 14 \\
\hline $3: 27: 02.1$ & $30: 15: 08$ & 10.4 & 0.13 & 0.6 & 7.8 & 1.3 & $4.2 \mathrm{E}+04$ & 19 \\
\hline $3: 27: 28.9$ & $30: 15: 04$ & 10.7 & 0.10 & 0.7 & 7.3 & 1.5 & $5.1 \mathrm{E}+04$ & 20 \\
\hline $3: 27: 40.0$ & $30: 12: 13$ & 10.5 & 0.17 & 0.4 & 18.6 & 0.9 & $1.6 \mathrm{E}+05$ & 23 \\
\hline $3: 27: 55.9$ & $30: 06: 18$ & 9.1 & 0.05 & 0.7 & 18.9 & 1.9 & $1.9 \mathrm{E}+05$ & 24 \\
\hline $3: 28: 05.5$ & $30: 06: 19$ & 9.4 & 0.11 & 0.7 & 17.7 & 1.8 & $1.8 \mathrm{E}+05$ & 24 \\
\hline $3: 28: 32.4$ & $31: 04: 43$ & 10.6 & 0.13 & 0.5 & 7.6 & 1.1 & $7.1 \mathrm{E}+04$ & 26 \\
\hline $3: 28: 33.4$ & $30: 19: 35$ & 10.7 & 0.12 & 1.0 & 10.0 & 2.2 & $3.7 \mathrm{E}+04$ & 27 \\
\hline $3: 28: 39.5$ & $31: 18: 35$ & 11.7 & 0.17 & 1.9 & 21.2 & 3.6 & $2.3 \mathrm{E}+05$ & 31 \\
\hline $3: 28: 48.5$ & $31: 16: 03$ & 11.3 & 0.10 & 0.2 & 7.6 & 0.4 & $1.8 \mathrm{E}+05$ & 35 \\
\hline $3: 28: 52.2$ & $31: 18: 08$ & 13.8 & 0.17 & 0.4 & 6.8 & 0.5 & $1.4 \mathrm{E}+05$ & 37 \\
\hline $3: 29: 18.5$ & $31: 25: 13$ & 11.9 & 0.12 & 1.4 & 18.1 & 2.5 & $1.4 \mathrm{E}+05$ & 53 \\
\hline $3: 29: 22.5$ & $31: 36: 24$ & 9.8 & 0.12 & 0.6 & 7.6 & 1.5 & $4.6 \mathrm{E}+04$ & 56 \\
\hline $3: 29: 25.8$ & $31: 28: 17$ & 10.3 & 0.11 & 0.3 & 18.8 & 0.8 & $6.3 \mathrm{E}+05$ & 58 \\
\hline $3: 30: 24.1$ & $30: 27: 39$ & 10.5 & 0.07 & 0.4 & 7.0 & 0.8 & $4.2 \mathrm{E}+04$ & 61 \\
\hline $3: 30: 45.6$ & $30: 52: 36$ & 10.5 & 0.13 & 0.7 & 9.4 & 1.5 & $5.5 \mathrm{E}+04$ & 63 \\
\hline $3: 30: 50.5$ & $30: 49: 17$ & 9.8 & 0.08 & 0.2 & 6.4 & 0.5 & $9.9 \mathrm{E}+04$ & 64 \\
\hline $3: 32: 26.9$ & $30: 59: 11$ & 10.5 & 0.13 & 0.9 & 11.0 & 1.9 & $1.0 \mathrm{E}+05$ & 67 \\
\hline $3: 32: 44.1$ & 31:00:01 & 10.2 & 0.11 & 2.0 & 16.7 & 4.6 & $9.2 \mathrm{E}+04$ & 70 \\
\hline $3: 32: 51.3$ & $31: 01: 48$ & 11.2 & 0.22 & 1.1 & 10.7 & 2.3 & $7.2 \mathrm{E}+04$ & 71 \\
\hline $3: 32: 57.0$ & $31: 03: 21$ & 10.1 & 0.12 & 0.9 & 16.8 & 2.2 & $1.6 \mathrm{E}+05$ & 72 \\
\hline $3: 33: 02.0$ & $31: 04: 33$ & 10.0 & 0.14 & 0.4 & 18.8 & 0.9 & $1.8 \mathrm{E}+05$ & 74 \\
\hline $3: 33: 04.3$ & $31: 04: 57$ & 9.9 & 0.18 & 0.4 & 19.2 & 0.9 & $1.8 \mathrm{E}+05$ & 75 \\
\hline $3: 33: 06.3$ & $31: 06: 26$ & 10.3 & 0.15 & 0.4 & 17.7 & 0.9 & $1.6 \mathrm{E}+05$ & 75 \\
\hline $3: 33: 25.2$ & $31: 05: 35$ & 9.7 & 0.10 & 0.4 & 11.1 & 0.9 & $1.5 \mathrm{E}+05$ & 82 \\
\hline $3: 40: 49.5$ & $31: 48: 35$ & 12.4 & 0.08 & 1.0 & 7.6 & 1.7 & $3.5 \mathrm{E}+04$ & 89 \\
\hline $3: 41: 40.2$ & $31: 58: 05$ & 9.5 & 0.07 & 0.2 & 7.5 & 0.6 & $8.7 \mathrm{E}+04$ & 92 \\
\hline $3: 41: 46.0$ & $31: 57: 22$ & 9.6 & 0.07 & 0.3 & 10.2 & 0.8 & $1.0 \mathrm{E}+05$ & 94 \\
\hline $3: 43: 44.0$ & $32: 02: 52$ & 12.9 & 0.13 & 0.8 & 11.7 & 1.3 & $1.5 \mathrm{E}+05$ & 100 \\
\hline $3: 43: 45.5$ & $32: 01: 44$ & 14.0 & 0.10 & 0.3 & 5.3 & 0.4 & $8.0 \mathrm{E}+04$ & 101 \\
\hline $3: 43: 45.8$ & $32: 03: 11$ & 11.7 & 0.16 & 0.8 & 13.8 & 1.5 & $1.7 \mathrm{E}+05$ & 100 \\
\hline $3: 43: 57.8$ & $32: 04: 06$ & 12.9 & 0.16 & 0.6 & 13.3 & 1.0 & $1.6 \mathrm{E}+05$ & 105 \\
\hline $3: 44: 05.1$ & $32: 00: 28$ & 10.7 & 0.20 & 0.4 & 8.2 & 0.8 & $9.4 \mathrm{E}+04$ & 109 \\
\hline $3: 44: 05.3$ & $32: 02: 05$ & 10.9 & 0.14 & 0.4 & 16.0 & 0.9 & $1.7 \mathrm{E}+05$ & 110 \\
\hline $3: 44: 14.6$ & $31: 57: 59$ & 10.6 & 0.12 & 0.8 & 11.1 & 1.8 & $6.9 \mathrm{E}+04$ & 111 \\
\hline $3: 44: 36.4$ & $31: 58: 40$ & 10.8 & 0.15 & 0.5 & 10.5 & 1.1 & $1.1 \mathrm{E}+05$ & 115 \\
\hline $3: 44: 48.8$ & $32: 00: 29$ & 10.8 & 0.10 & 0.4 & 9.4 & 0.8 & $1.6 \mathrm{E}+05$ & 117 \\
\hline $3: 44: 56.1$ & $32: 00: 32$ & 10.9 & 0.12 & 0.3 & 6.8 & 0.7 & $7.4 \mathrm{E}+04$ & 118 \\
\hline $3: 45: 15.9$ & $32: 04: 49$ & 10.7 & 0.08 & 1.3 & 11.6 & 2.8 & $4.9 \mathrm{E}+04$ & 119 \\
\hline $3: 47: 33.5$ & $32: 50: 55$ & 10.0 & 0.08 & 0.7 & 9.9 & 1.6 & $5.0 \mathrm{E}+04$ & 121 \\
\hline $3: 47: 38.6$ & $32: 52: 19$ & 10.1 & 0.17 & 1.3 & 18.5 & 3.2 & $1.8 \mathrm{E}+05$ & 122 \\
\hline $3: 47: 39.7$ & $32: 53: 57$ & 10.1 & 0.10 & 1.3 & 18.5 & 3.2 & $1.8 \mathrm{E}+05$ & 122 \\
\hline $3: 47: 39.8$ & $32: 53: 34$ & 10.0 & 0.11 & 1.3 & 18.8 & 3.2 & $1.8 \mathrm{E}+05$ & 122 \\
\hline
\end{tabular}

Notes.

a $\mathrm{J} 2000$ position of $\mathrm{NH}_{3}$ pointing.

b $\mathrm{J} 2000$ position of $\mathrm{NH}_{3}$ pointing.

${ }^{\mathrm{c}}$ Kinetic temperature of core derived from $\mathrm{NH}_{3}(1,1)$ and $(2,2)$ spectra.

${ }^{\mathrm{d}}$ Nonthermal one-dimensional velocity dispersion of $\mathrm{NH}_{3}$ spectra.

e Total $1.1 \mathrm{~mm}$ flux of the Bolocam core.

${ }^{\mathrm{f}}$ Peak column density of the core derived from the peak $1.1 \mathrm{~mm}$ flux and $T_{\text {kin }}$.

g Total mass of Bolocam core derived from the integrated $1.1 \mathrm{~mm}$ flux and $T_{\text {kin }}$.

${ }^{\mathrm{h}}$ Average density of free particles of Bolocam core derived from its mass and size.

i Per-Bolo number taken from Table 4 in Enoch et al. (2008a).

estimate the line-of-sight averaged temperature of the dust $\left(T_{\mathrm{d}}\right.$; Schnee et al. 2008) and the gas ( $T_{\mathrm{CO}}$; Pineda et al. 2008) in the
Perseus molecular cloud and compare it with the temperature derived from the $\mathrm{NH}_{3}$ pointings. The resolution of the $T_{\mathrm{d}}$ and 
Table 2

Environment of Starless Cores

\begin{tabular}{|c|c|c|c|c|c|c|c|c|c|}
\hline $\begin{array}{l}\text { R.A. } \\
\text { J2000 }\end{array}$ & $\begin{array}{l}\text { Decl. }^{\mathrm{b}} \\
\text { J2000 }\end{array}$ & $\begin{array}{c}T_{\text {kin }}{ }^{c} \\
(\mathrm{~K})\end{array}$ & $\begin{array}{l}T_{\mathrm{d}}^{\mathrm{d}} \\
(\mathrm{K})\end{array}$ & $\begin{array}{c}T_{\mathrm{CO}}{ }^{\mathrm{e}} \\
(\mathrm{K})\end{array}$ & $\begin{array}{c}\mathrm{NIR} A_{V}{ }^{\mathrm{f}} \\
(\mathrm{mag})\end{array}$ & $\begin{array}{c}\text { FIR } A_{V} \mathrm{~g} \\
(\mathrm{mag})\end{array}$ & $\begin{array}{c}\sigma_{\mathrm{CO}^{\mathrm{h}}} \\
\left(\mathrm{km} \mathrm{s}^{-1}\right)\end{array}$ & Distance Class $0 / \mathrm{I}^{\mathrm{i}}$ & In a Cluster? ${ }^{j}$ \\
\hline $3: 25: 07.8$ & $30: 24: 22$ & 9.2 & 14.6 & 11.6 & 5.0 & 2.6 & 0.5 & 1.51 & $\mathrm{~N}$ \\
\hline $3: 25: 09.7$ & $30: 23: 53$ & 9.2 & 14.6 & 11.2 & 5.0 & 2.6 & 0.6 & 1.50 & $\mathrm{~N}$ \\
\hline $3: 25: 10.1$ & $30: 44: 41$ & 10.0 & 15.2 & 12.9 & 5.1 & 4.0 & 0.6 & 0.19 & $\mathrm{~N}$ \\
\hline $3: 25: 17.1$ & $30: 18: 53$ & 10.3 & 12.9 & 12.0 & 5.0 & 7.2 & 0.7 & 1.28 & $\mathrm{~N}$ \\
\hline $3: 25: 26.9$ & $30: 21: 53$ & 9.1 & 12.9 & 12.7 & 3.7 & 6.7 & 0.5 & 1.20 & $\mathrm{~N}$ \\
\hline $3: 25: 46.1$ & $30: 44: 11$ & 11.2 & 15.5 & 11.0 & 5.5 & 5.1 & 0.7 & 0.12 & $\mathrm{~N}$ \\
\hline $3: 25: 48.8$ & $30: 42: 24$ & 9.1 & 14.3 & 11.2 & 4.8 & 5.4 & 0.7 & 0.20 & $\mathrm{~N}$ \\
\hline $3: 25: 50.6$ & $30: 42: 02$ & 9.0 & 12.5 & 10.6 & 4.8 & 10.4 & 0.7 & 0.24 & $\mathrm{~N}$ \\
\hline $3: 27: 02.1$ & $30: 15: 08$ & 10.4 & 13.5 & 13.1 & 4.8 & 3.8 & 0.6 & 0.39 & $\mathrm{~N}$ \\
\hline $3: 27: 28.9$ & $30: 15: 04$ & 10.7 & 14.9 & $\mathrm{NaN}$ & 9.5 & 2.9 & $\mathrm{NaN}$ & 0.16 & $\mathrm{~N}$ \\
\hline $3: 27: 40.0$ & $30: 12: 13$ & 10.5 & 17.4 & $\mathrm{NaN}$ & 8.8 & 2.8 & $\mathrm{NaN}$ & 0.04 & $\mathrm{~N}$ \\
\hline $3: 27: 55.9$ & $30: 06: 18$ & 9.1 & 13.7 & $\mathrm{NaN}$ & 3.1 & 3.1 & $\mathrm{NaN}$ & 0.17 & $\mathrm{~N}$ \\
\hline $3: 28: 05.5$ & $30: 06: 19$ & 9.4 & 13.7 & $\mathrm{NaN}$ & 2.9 & 3.1 & $\mathrm{NaN}$ & 0.16 & $\mathrm{~N}$ \\
\hline $3: 28: 32.4$ & $31: 04: 43$ & 10.6 & 12.8 & 18.8 & 5.4 & 8.3 & 1.1 & 0.14 & $\mathrm{Y}$ \\
\hline $3: 28: 33.4$ & $30: 19: 35$ & 10.7 & 13.0 & $\mathrm{NaN}$ & 4.5 & 6.0 & $\mathrm{NaN}$ & 0.90 & $\mathrm{~N}$ \\
\hline $3: 28: 39.5$ & $31: 18: 35$ & 11.7 & 12.3 & 18.6 & 7.8 & 19.1 & 0.8 & 0.05 & $\mathrm{Y}$ \\
\hline $3: 28: 48.5$ & $31: 16: 03$ & 11.3 & 16.0 & 16.2 & 8.5 & 0.0 & 0.9 & 0.15 & $\mathrm{Y}$ \\
\hline $3: 28: 52.2$ & $31: 18: 08$ & 13.8 & 15.0 & 19.4 & 9.2 & 10.7 & 1.3 & 0.10 & $\mathrm{Y}$ \\
\hline $3: 29: 18.5$ & $31: 25: 13$ & 11.9 & $\mathrm{NaN}$ & 49.0 & 6.5 & $\mathrm{NaN}$ & 0.7 & 0.13 & $\mathrm{Y}$ \\
\hline $3: 29: 22.5$ & $31: 36: 24$ & 9.8 & 15.2 & 15.5 & 5.9 & 2.9 & 0.5 & 0.23 & $\mathrm{~N}$ \\
\hline $3: 29: 25.8$ & $31: 28: 17$ & 10.3 & 15.6 & 23.3 & 5.5 & 0.0 & 1.2 & 0.14 & $\mathrm{Y}$ \\
\hline $3: 30: 24.1$ & $30: 27: 39$ & 10.5 & 14.2 & $\mathrm{NaN}$ & 7.0 & 5.5 & $\mathrm{NaN}$ & 0.16 & $\mathrm{~N}$ \\
\hline $3: 30: 45.6$ & $30: 52: 36$ & 10.5 & 13.3 & 12.4 & 2.7 & 4.8 & 0.9 & 0.74 & $\mathrm{~N}$ \\
\hline $3: 30: 50.5$ & $30: 49: 17$ & 9.8 & 13.5 & 11.6 & 2.1 & 2.8 & 0.8 & 0.54 & $\mathrm{~N}$ \\
\hline $3: 32: 26.9$ & $30: 59: 11$ & 10.5 & 13.4 & 13.5 & 10.3 & 9.0 & 0.7 & 0.23 & $\mathrm{~N}$ \\
\hline $3: 32: 44.1$ & 31:00:01 & 10.2 & 12.2 & 12.8 & 10.0 & 21.7 & 0.9 & 0.30 & $\mathrm{~N}$ \\
\hline $3: 32: 51.3$ & $31: 01: 48$ & 11.2 & 12.3 & 13.7 & 8.8 & 20.3 & 0.9 & 0.36 & $\mathrm{~N}$ \\
\hline $3: 32: 57.0$ & $31: 03: 21$ & 10.1 & 12.4 & 14.4 & 8.5 & 15.4 & 0.8 & 0.39 & $\mathrm{~N}$ \\
\hline $3: 33: 02.0$ & $31: 04: 33$ & 10.0 & 12.5 & 13.9 & 8.5 & 16.7 & 0.9 & 0.27 & $\mathrm{~N}$ \\
\hline $3: 33: 04.3$ & $31: 04: 57$ & 9.9 & 12.5 & 14.0 & 8.5 & 15.3 & 0.8 & 0.23 & $\mathrm{~N}$ \\
\hline $3: 33: 06.3$ & $31: 06: 26$ & 10.3 & 12.7 & 13.3 & 8.5 & 13.3 & 0.9 & 0.14 & $\mathrm{~N}$ \\
\hline $3: 33: 25.2$ & $31: 05: 35$ & 9.7 & 12.5 & 15.8 & 8.6 & 19.1 & 0.9 & 0.10 & $\mathrm{~N}$ \\
\hline $3: 40: 49.5$ & $31: 48: 35$ & 12.4 & 16.1 & 15.4 & 7.7 & 9.1 & 0.9 & 0.42 & $\mathrm{~N}$ \\
\hline $3: 41: 40.2$ & $31: 58: 05$ & 9.5 & 16.3 & 21.3 & 8.4 & 7.5 & 0.6 & 1.09 & $\mathrm{~N}$ \\
\hline $3: 41: 46.0$ & $31: 57: 22$ & 9.6 & 15.6 & 19.7 & 8.4 & 10.3 & 0.7 & 1.09 & $\mathrm{~N}$ \\
\hline $3: 43: 44.0$ & $32: 02: 52$ & 12.9 & $\mathrm{NaN}$ & 21.8 & 9.5 & $\mathrm{NaN}$ & 0.6 & 0.11 & $\mathrm{Y}$ \\
\hline $3: 43: 45.5$ & $32: 01: 44$ & 14.0 & 14.6 & 20.7 & 10.1 & 15.8 & 0.5 & 0.14 & $\mathrm{Y}$ \\
\hline $3: 43: 45.8$ & $32: 03: 11$ & 11.7 & 15.1 & 21.4 & 8.7 & 21.3 & 0.6 & 0.07 & $\mathrm{Y}$ \\
\hline $3: 43: 57.8$ & $32: 04: 06$ & 12.9 & 15.4 & 27.0 & 7.4 & 19.0 & 0.8 & 0.08 & $\mathrm{Y}$ \\
\hline $3: 44: 05.1$ & $32: 00: 28$ & 10.7 & 14.7 & 21.9 & 9.0 & 14.8 & 0.8 & 0.13 & $\mathrm{Y}$ \\
\hline $3: 44: 05.3$ & $32: 02: 05$ & 10.9 & $\mathrm{NaN}$ & 23.5 & 9.0 & $\mathrm{NaN}$ & 0.6 & 0.06 & $\mathrm{Y}$ \\
\hline $3: 44: 14.6$ & $31: 57: 59$ & 10.6 & $\mathrm{NaN}$ & 17.0 & 7.9 & $\mathrm{NaN}$ & 0.7 & 0.16 & $\mathrm{Y}$ \\
\hline $3: 44: 36.4$ & $31: 58: 40$ & 10.8 & 15.3 & 16.8 & 7.2 & 9.1 & 0.8 & 0.22 & Y \\
\hline $3: 44: 48.8$ & $32: 00: 29$ & 10.8 & 14.8 & 16.9 & 5.9 & 12.5 & 0.6 & 0.10 & $\mathrm{Y}$ \\
\hline $3: 44: 56.1$ & $32: 00: 32$ & 10.9 & 14.9 & 18.6 & 5.4 & 10.0 & 0.5 & 0.20 & $\mathrm{Y}$ \\
\hline $3: 45: 15.9$ & $32: 04: 49$ & 10.7 & 14.3 & 24.5 & 5.2 & 12.3 & 0.5 & 0.55 & $\mathrm{Y}$ \\
\hline $3: 47: 33.5$ & $32: 50: 55$ & 10.0 & 14.0 & 16.5 & 5.8 & 6.5 & 0.5 & 0.14 & $\mathrm{~N}$ \\
\hline $3: 47: 38.6$ & $32: 52: 19$ & 10.1 & 13.4 & 16.0 & 7.6 & 10.7 & 0.7 & 0.06 & $\mathrm{~N}$ \\
\hline $3: 47: 39.7$ & $32: 53: 57$ & 10.1 & 12.7 & 14.2 & 7.6 & 11.9 & 0.6 & 0.16 & $\mathrm{~N}$ \\
\hline $3: 47: 39.8$ & $32: 53: 34$ & 10.0 & 12.4 & 14.8 & 7.6 & 14.8 & 0.6 & 0.13 & $\mathrm{~N}$ \\
\hline
\end{tabular}

Notes.

a J2000 position of $\mathrm{NH}_{3}$ pointing.

b $\mathrm{J} 2000$ position of $\mathrm{NH}_{3}$ pointing.

${ }^{c}$ Kinetic temperature of core derived from $\mathrm{NH}_{3}(1,1)$ and $(2,2)$ spectra.

${ }^{\mathrm{d}}$ Dust temperature of the Perseus molecular cloud derived from FIR emission.

${ }^{\mathrm{e}}$ Gas temperature of the Perseus molecular cloud derived from ${ }^{12} \mathrm{CO}(1-0)$ emission.

${ }^{\mathrm{f}}$ Column density of the Perseus molecular cloud derived from NIR extinction.

g Column density of the Perseus molecular cloud derived from FIR emission.

${ }^{\mathrm{h}}$ One-dimensional velocity dispersion of ${ }^{13} \mathrm{CO}(1-0)$.

${ }^{i}$ Distance to the nearest Class 0/I protostar.

${ }^{\mathrm{j}}$ Is the core within a cluster (NGC1333 or IC348)? 


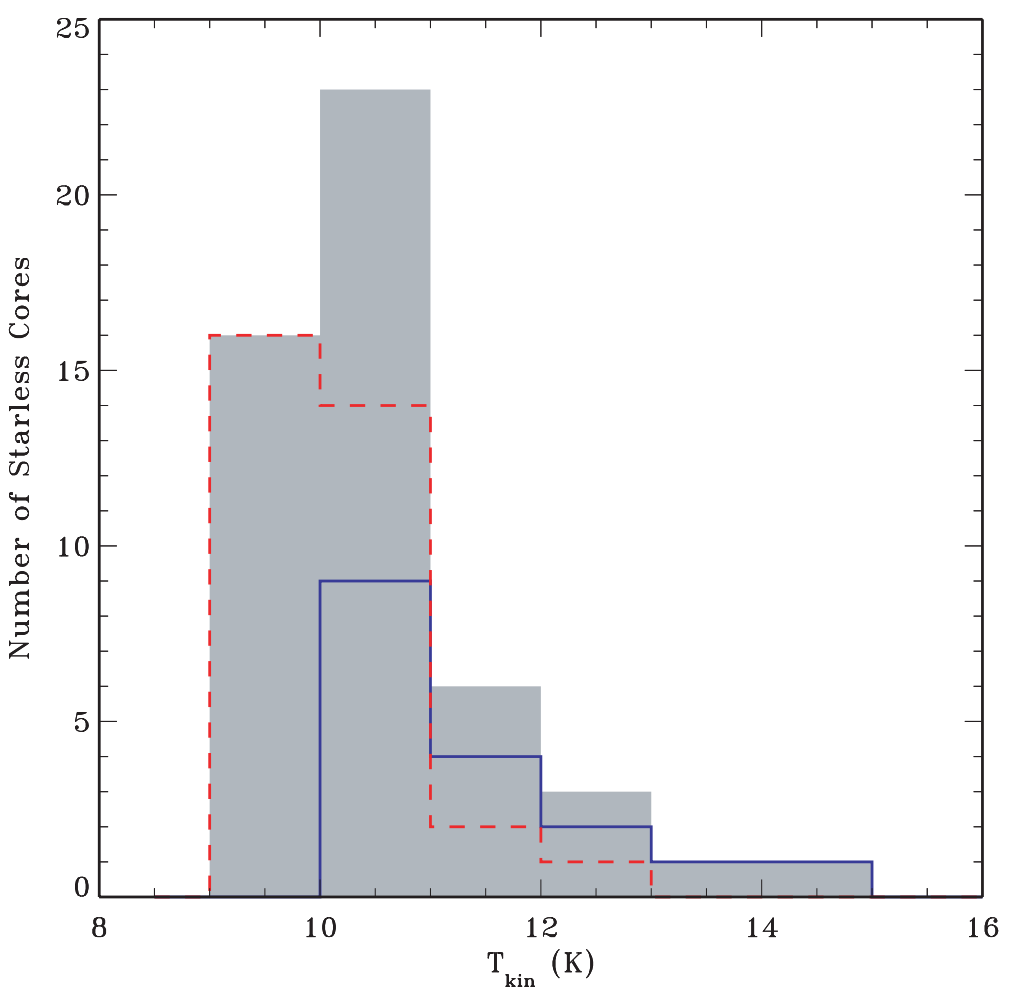

Figure 1. Histogram of $T_{\text {kin }}$. The filled (gray) histogram is the temperature distribution of all 50 of the starless cores in Perseus for which we have well fit $\mathrm{NH}_{3}$ spectra. The dashed (red) and solid (blue) histograms are the temperature distributions of the starless cores in the field and inside the clusters, respectively.

(A color version of this figure is available in the online journal.)

$T_{\mathrm{CO}}$ maps are both $\sim 40^{\prime \prime}$, which is roughly comparable to the $31^{\prime \prime}$ resolution of the ammonia data. However, both $T_{\mathrm{d}}$ and $T_{\mathrm{CO}}$ are imperfect measures of cloud temperature. Because ${ }^{12} \mathrm{CO}$ is optically thick (and perhaps depleted), it is not sensitive to the colder and denser cloud material in which the starless cores are embedded. The dust temperature determination is emissionweighted, and at the FIR wavelengths (70 and $160 \mu \mathrm{m})$ of the Spitzer maps the emission is dominated by warm dust along the line of sight, and therefore is less sensitive to the colder and denser clumps in which cores form.

Nevertheless, one might expect that the temperature of a starless core could be affected, or predicted, by the temperature of the medium in which is it embedded. Starless cores are, by definition, externally heated, so whatever is heating the cloud should also be heating the embedded cores. This analysis can be biased because the gas- and dust-derived temperatures are warmer within the clusters than outside, which is also true for $T_{\mathrm{kin}}$, so any derived correlations must take this into account. Figure 2 and Table 3 show that although both $T_{\mathrm{CO}}$ and $T_{\mathrm{d}}$ are significantly and positively correlated with $T_{\mathrm{kin}}$ when considering the entire population of starless cores, this effect is entirely explained by the cluster/field bias, and within each subpopulation there is no significant correlation.

\section{4. $T_{\text {kin }}$ versus Column Density}

The heating of starless cores is governed by the strength of the ISRF. Given that there are no O or B stars within the Perseus molecular cloud, the ISRF seen by the cores should depend largely on dust shielding, but not on the distance to any special source of external radiation. Because we are unable to measure the volume-averaged shielding around each starless core, we use as a proxy the column density along the line of sight. Based on this simple analysis, one would predict that $T_{\text {kin }}$ would be anticorrelated with column density.

On the other hand, for starless cores that are in pressure equilibrium, the inward pressures due to the weight of the Perseus molecular cloud and the self-gravity of the cores will be counterbalanced by the internal gas pressure from thermal and nonthermal motions. The external pressure on the starless cores from the molecular cloud is proportional to the square of the column density $\left(P_{\text {cloud }} \propto A_{V}^{2}\right.$; Bertoldi \& McKee 1992; Lada et al. 2008), and the internal gas pressure from thermal motions is proportional to $T_{\text {kin }}\left(P_{\text {thermal }} \propto \sigma_{\mathrm{T}}^{2} \propto T_{\text {kin }}\right)$. From a balance of pressure analysis, one would expect a positive correlation between $T_{\mathrm{kin}}$ and column density, though this would not necessarily be a strong effect because outward pressure is also supplied by nonthermal motions and magnetic fields. We calculate that for the starless cores in our sample, the inward pressure of the weight of the molecular cloud is roughly a factor of 2 weaker than their self-gravity.

Here we compare the temperatures of starless cores with the column density of molecular cloud material along the line of sight to each core. We estimate the cloud column density using FIR dust emission (Schnee et al. 2008) and NIR extinction (Ridge et al. 2006). Although extinction may be the most secure estimator of column density in a molecular cloud (Goodman et al. 2008), the $40^{\prime \prime}$ resolution of the emission-derived column density map compares favorably with the $5^{\prime}$ resolution of the NIR extinction map. As with the cloud temperature, there is an inherent bias in that the column density in the clusters is higher and the core temperatures are higher, so we consider the field and cluster starless cores separately. Figure 2 and Table 3 show that there is a significant positive correlation between extinction-derived column density and $T_{\text {kin }}$ when considering just the starless cores in the field and just the starless cores in 

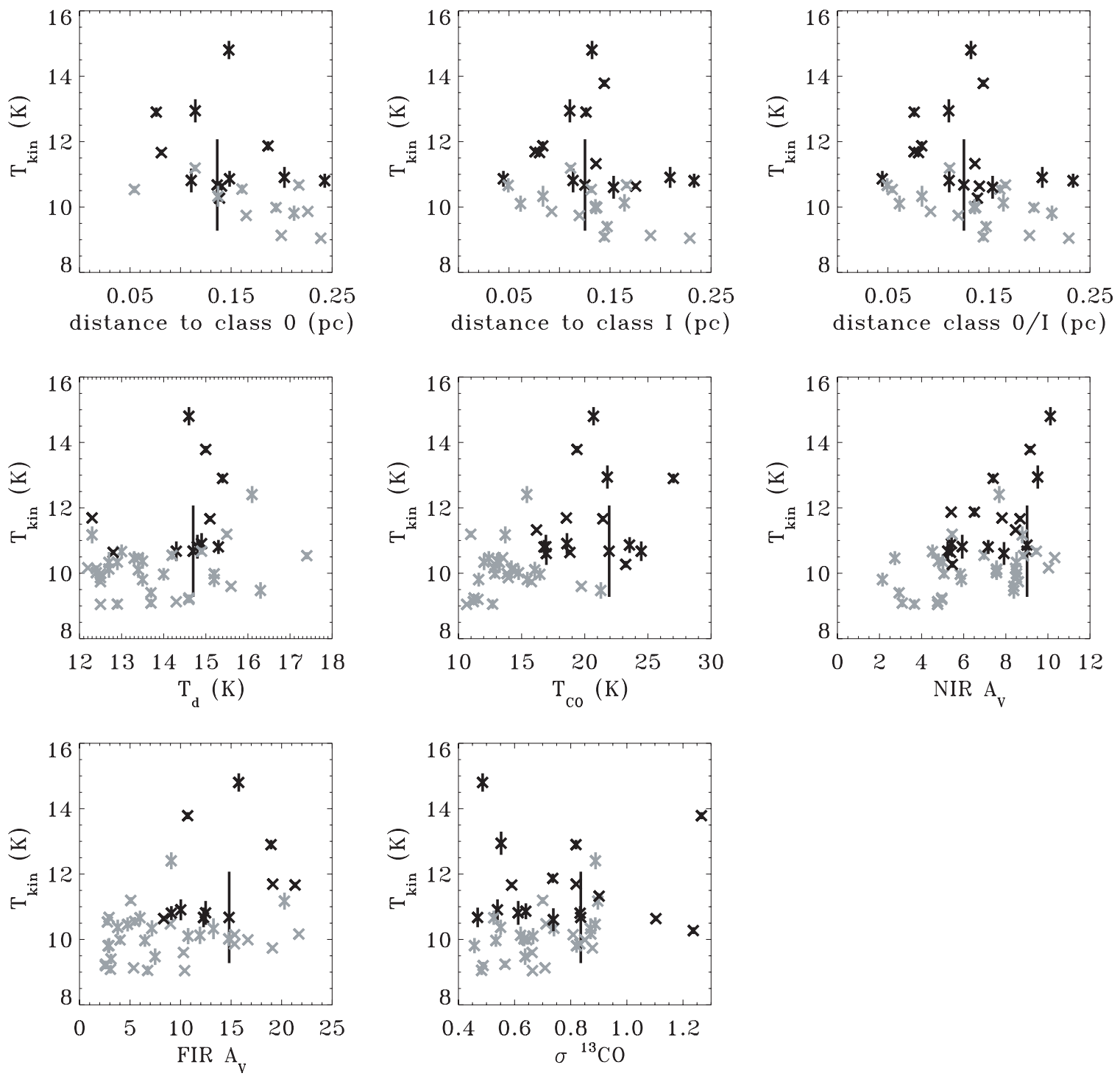

Figure 2. $T_{\text {kin }}$ plotted against properties of the environment around the starless cores: (top left) distance to the nearest Class 0 protostar; (top middle) distance to the nearest Class I protostar; (top right) distance to the nearest Class 0 or Class I protostar; (middle left) dust temperature; (center) gas temperature; (middle right) column density of the molecular cloud derived from NIR extinction; (bottom left) column density of the cloud derived from FIR emission; and (bottom middle) ${ }^{13} \mathrm{CO}$ line width (one-dimensional velocity dispersion). In this plot, Class 0 cores and Class I cores are identified as in Enoch et al. (2008b). Starless cores in NGC1333 and IC348 are plotted in black, and field starless cores are plotted in gray. The $1 \sigma$ errors in the fit of $T_{\text {kin }}$ are plotted for each core.

the clusters, as well as for the field+cluster ensemble. There is no significant trend between $T_{\text {kin }}$ and the dust emission-derived column density for field or cluster cores.

One should keep in mind that the emission-derived column density derived from dust emission loses accuracy at values of $A_{V}>$ a few, so it is not so surprising to get different results depending on how the column density is estimated (Schnee et al. 2006). The dust temperature derived from FIR maps is emission-weighted, and the column density derivation depends on the dust temperature, so the column density along lines of sight with significant variations in temperature and density is both systemically underestimated and subject to large scatter. This effect could wash out any true correlations between the column density of the cloud material and the temperature of the core embedded in it.

In a numerical simulation of embedded starless cores, Stamatellos \& Whitworth (2003) show that the temperature profile of a core shielded by $A_{V}=5$ material (a typical value for $A_{V}$ seen toward the starless cores in Perseus) is not significantly different from a naked core, while deeply embedded cores $\left(A_{V}=20\right)$ are noticeably colder than naked cores. In addition, we expect that the column density along the line of sight is a poor indicator of volumetric shielding due to the porosity of molecular clouds like Perseus. The visual impression given by two-dimensional maps and position-position-velocity cubes of nearby molecular clouds is one of the nonrelaxed structures with many filaments, voids, and shells, supporting this view. The observational evidence supports the idea that the shielding provided by higher $A_{V}$ material in Perseus does not lead to lower temperatures of the embedded starless cores. The opposite effect is observed, which may be explained by the greater thermal support needed to balance the increased pressure on the cores by the weight of the molecular cloud.

\section{5. $T_{k i n}$ versus $\sigma_{\mathrm{NT}}$ and $\sigma_{\mathrm{CO}}$}

Clumpiness formed by turbulence in a molecular cloud is known to significantly affect the temperature of the cloud 
Table 3

$T_{\text {kin }}$ Correlations

\begin{tabular}{|c|c|c|c|c|c|}
\hline Property & Location & $r$ Value $^{\mathrm{a}}$ & $t$ Value $^{\mathrm{b}}$ & Confidence $^{\mathrm{c}}$ & $\mathrm{DOF}^{\mathrm{d}}$ \\
\hline$T_{\mathrm{d}}$ & Field+cluster & 0.27 & 1.83 & 96.3 & 44 \\
\hline$T_{\mathrm{CO}}$ & Field+cluster & 0.45 & 3.26 & $>99.9$ & 42 \\
\hline NIR $A_{V}$ & Field+cluster & 0.47 & 3.66 & $>99.9$ & 48 \\
\hline FIR $A_{V}$ & Field+cluster & 0.34 & 2.43 & 99.0 & 44 \\
\hline$\sigma_{\mathrm{CO}}$ & Field+cluster & 0.20 & 1.31 & 90.1 & 42 \\
\hline$\sigma_{\mathrm{NT}}$ & Field+cluster & 0.28 & 2.06 & 97.8 & 48 \\
\hline $1.1 \mathrm{~mm}$ flux & Field+cluster & 0.11 & 0.73 & 76.6 & 48 \\
\hline Peak $A_{V}$ & Field+cluster & -0.33 & 2.39 & 99.0 & 48 \\
\hline Mass & Field+cluster & -0.11 & 0.78 & 78.0 & 48 \\
\hline Density & Field+cluster & -0.18 & 1.25 & 89.1 & 48 \\
\hline Dist. $0^{\mathrm{e}}$ & Field+cluster & -0.45 & 2.29 & 98.3 & 21 \\
\hline Dist. $\mathrm{I}^{\mathrm{e}}$ & Field+cluster & -0.21 & 1.14 & 86.8 & 29 \\
\hline Dist. $0 / \mathrm{I}^{\mathrm{e}}$ & Field+cluster & -0.29 & 1.71 & 95.1 & 33 \\
\hline$T_{\mathrm{d}}$ & Field only & 0.16 & 0.93 & 82.0 & 31 \\
\hline$T_{\mathrm{CO}}$ & Field only & 0.04 & 0.20 & 57.8 & 25 \\
\hline $\mathrm{NIR} A_{V}$ & Field only & 0.36 & 2.17 & 98.1 & 31 \\
\hline FIR $A_{V}$ & Field only & 0.16 & 0.91 & 81.5 & 31 \\
\hline$\sigma_{\mathrm{CO}}$ & Field only & 0.50 & 2.90 & 99.6 & 25 \\
\hline$\sigma_{\mathrm{NT}}$ & Field only & 0.27 & 1.55 & 93.5 & 31 \\
\hline $1.1 \mathrm{~mm}$ flux & Field only & 0.35 & 2.06 & 97.6 & 31 \\
\hline Peak $A_{V}$ & Field only & -0.36 & 2.17 & 98.1 & 31 \\
\hline Mass & Field only & 0.15 & 0.86 & 80.1 & 31 \\
\hline Density & Field only & -0.54 & 3.58 & $>99.1$ & 31 \\
\hline Dist. $0^{\mathrm{e}}$ & Field only & -0.61 & 2.32 & 97.7 & 9 \\
\hline Dist. $\mathrm{I}^{\mathrm{e}}$ & Field only & -0.54 & 2.43 & 98.6 & 14 \\
\hline Dist. $0 / \mathrm{I}^{\mathrm{e}}$ & Field only & -0.51 & 2.42 & 98.7 & 17 \\
\hline$T_{\mathrm{d}}$ & Cluster only & 0.06 & 0.19 & 57.4 & 11 \\
\hline$T_{\mathrm{CO}}$ & Cluster only & 0.12 & 0.46 & 67.4 & 15 \\
\hline $\mathrm{NIR} A_{V}$ & Cluster only & 0.63 & 3.13 & 99.7 & 15 \\
\hline FIR $A_{V}$ & Cluster only & 0.39 & 1.41 & 90.7 & 11 \\
\hline$\sigma_{\mathrm{CO}}$ & Cluster only & -0.09 & 0.36 & 63.8 & 15 \\
\hline$\sigma_{\mathrm{NT}}$ & Cluster only & 0.14 & 0.56 & 70.8 & 15 \\
\hline $1.1 \mathrm{~mm}$ Flux & Cluster only & -0.03 & 0.12 & 54.7 & 15 \\
\hline Peak $A_{V}$ & Cluster only & -0.25 & 0.99 & 83.1 & 15 \\
\hline Mass & Cluster only & -0.19 & 0.76 & 77.0 & 15 \\
\hline Density & Cluster only & -0.19 & 0.75 & 76.8 & 15 \\
\hline Dist. $0^{\mathrm{e}}$ & Cluster only & -0.26 & 0.86 & 79.5 & 10 \\
\hline Dist. $\mathrm{I}^{\mathrm{e}}$ & Cluster only & -0.15 & 0.55 & 70.4 & 13 \\
\hline Dist. $0 / \mathrm{I}^{\mathrm{e}}$ & Cluster only & -0.18 & 0.69 & 74.9 & 14 \\
\hline
\end{tabular}

Notes.

${ }^{\text {a }}$ Coefficient of correlation $(r)$.

${ }^{\mathrm{b}} t$ statistic: $r / \operatorname{sqrt}\left[\left(1-r^{2}\right) / \mathrm{dof}\right]$.

${ }^{c}$ Percentage confidence of correlation.

${ }^{\mathrm{d}}$ Degrees of freedom.

e Distance to a Class 0/I source, as identified in Enoch et al. (2008b) and plotted in Figure 2.

material, with higher turbulence leading to greater porosity and penetration by the ISRF, and therefore higher temperatures (Bethell et al. 2004). However, Stamatellos et al. (2007) find that the densest portions of the cloud (where starless cores are found) are primarily heated by long wavelength radiation that is not effectively shielded by the clumpy structure. It is therefore not obvious if one would expect starless core temperatures to be correlated with turbulence.

Here we examine whether turbulence affects the temperature of the starless cores in the Perseus molecular cloud. We use the nonthermal line width of $\mathrm{NH}_{3}$ as a proxy for the turbulence within the cores, and the total line width of ${ }^{13} \mathrm{CO}$ as an estimate of the turbulence in the surrounding medium (the measured ${ }^{13} \mathrm{CO}$ line width is roughly a factor of 10 higher than the thermal line width). As before, we consider field and cluster starless cores separately to avoid confusing a correlation between $T_{\text {kin }}$ and turbulence with a correlation between $T_{\text {kin }}$ and the field versus cluster environment.

Figure 3 and Table 3 show that there is no correlation between the nonthermal line width of $\mathrm{NH}_{3}$ and $T_{\text {kin }}$ when considering the field and cluster populations of starless cores separately, but there is a significant positive correlation for the ensemble. This implies that those starless cores in Perseus are more turbulent when found in clusters than in the field, but the turbulence itself does not necessarily lead to higher temperatures. Our expectation of starless core geometry is that they are in some sense "relaxed" structures, and therefore not porous, regardless of the level of internal turbulence. CO line width does not correlate with $T_{\text {kin }}$ for those cores in clusters, but it is significantly positively correlated with $T_{\text {kin }}$ for field starless cores, as shown in Figure 2. One interpretation of the field/cluster difference is that within clusters, the CO line width is likely to be affected strongly by the ongoing star formation, while the $\mathrm{CO}$ line width in the field better traces the cloud geometry, so only in the field does a larger CO line width imply better penetration by the ISRF.

\section{6. $T_{\text {kin }}$ versus $1.1 \mathrm{~mm}$ Continuum-Derived Properties}

It might be supposed that the temperatures of starless cores are partly determined by the internal properties of the cores themselves, and not just by their environment. Here we compare $T_{\text {kin }}$ with $1.1 \mathrm{~mm}$ Bolocam flux, the peak column density (expressed in $A_{V}$ ), the mass and the average volume density derived from the $1.1 \mathrm{~mm}$ map, and the ammonia-derived temperature of each core.

The peak $A_{V}$ is derived from the peak $1.1 \mathrm{~mm}$ flux $\left(S_{v}^{\text {beam }}\right)$ of each core and the ammonia-derived temperature,

$$
N\left(\mathrm{H}_{2}\right)=\frac{S_{v}^{\text {beam }}}{\Omega_{\text {beam }} \mu_{\mathrm{H}_{2}} m_{\mathrm{H}} \kappa_{v} B_{v}\left(T_{\text {kin }}\right)},
$$

with $N\left(\mathrm{H}_{2}\right) / A_{V}=0.94 \times 10^{21} \mathrm{~cm}^{2} \mathrm{mag}^{-1}$ (Frerking et al. 1982). Here $\Omega_{\text {beam }}$ is the beam solid angle, $m_{\mathrm{H}}$ is the mass of hydrogen, $\kappa_{1.1 \mathrm{~mm}}=0.0114 \mathrm{~cm}^{2} \mathrm{~g}^{-1}$ is the dust opacity per gram of gas interpolated from Column 5 of Table 1 of Ossenkopf \& Henning (1994) for dust grains with thin ice mantles, $B_{v}$ is the Planck function, and $\mu_{\mathrm{H}_{2}}=2.8$ is the mean molecular weight per $\mathrm{H}_{2}$ molecule. A gas to dust mass ratio of 100 is included in $\kappa_{1.1 \mathrm{~mm}}$.

The core mass is derived from the total $1 \mathrm{~mm}$ flux $\left(S_{v}\right)$ and $T_{\text {kin }}$ using the equation,

$$
M=\frac{d^{2} S_{v}}{B_{v}\left(T_{\text {kin }}\right) \kappa_{v}},
$$

where $\kappa_{\nu}$ is the dust opacity, $d$ is the distance to Perseus (250 pc). The density is derived from the core mass and the deconvolved average FWHM size $(D)$ of the core $\left(n=6 M / \pi D^{3} \mu_{p}\right)$, where $\mu_{p}=2.33$ is the mean molecular weight per particle, as explained in Enoch et al. (2006).

When deriving the core mass, peak column density, and average volume density from the $1.1 \mathrm{~mm}$ continuum emission, we use the gas temperature $\left(T_{\text {kin }}\right)$ in the calculation, under the assumption that the gas and dust temperatures are coupled. Although this assumption is valid for the densities $(n>2 \times$ $10^{4} \mathrm{~cm}^{-3}$ ) derived for these cores (Goldsmith 2001; Galli et al. 2002), we are introducing a potential bias in our analysis because $T_{\text {kin }}$ and the derived mass are not independent. It would be preferable to use the $T_{\text {dust }}$ rather than $T_{\text {kin }}$ to determine the $1.1 \mathrm{~mm}$ 

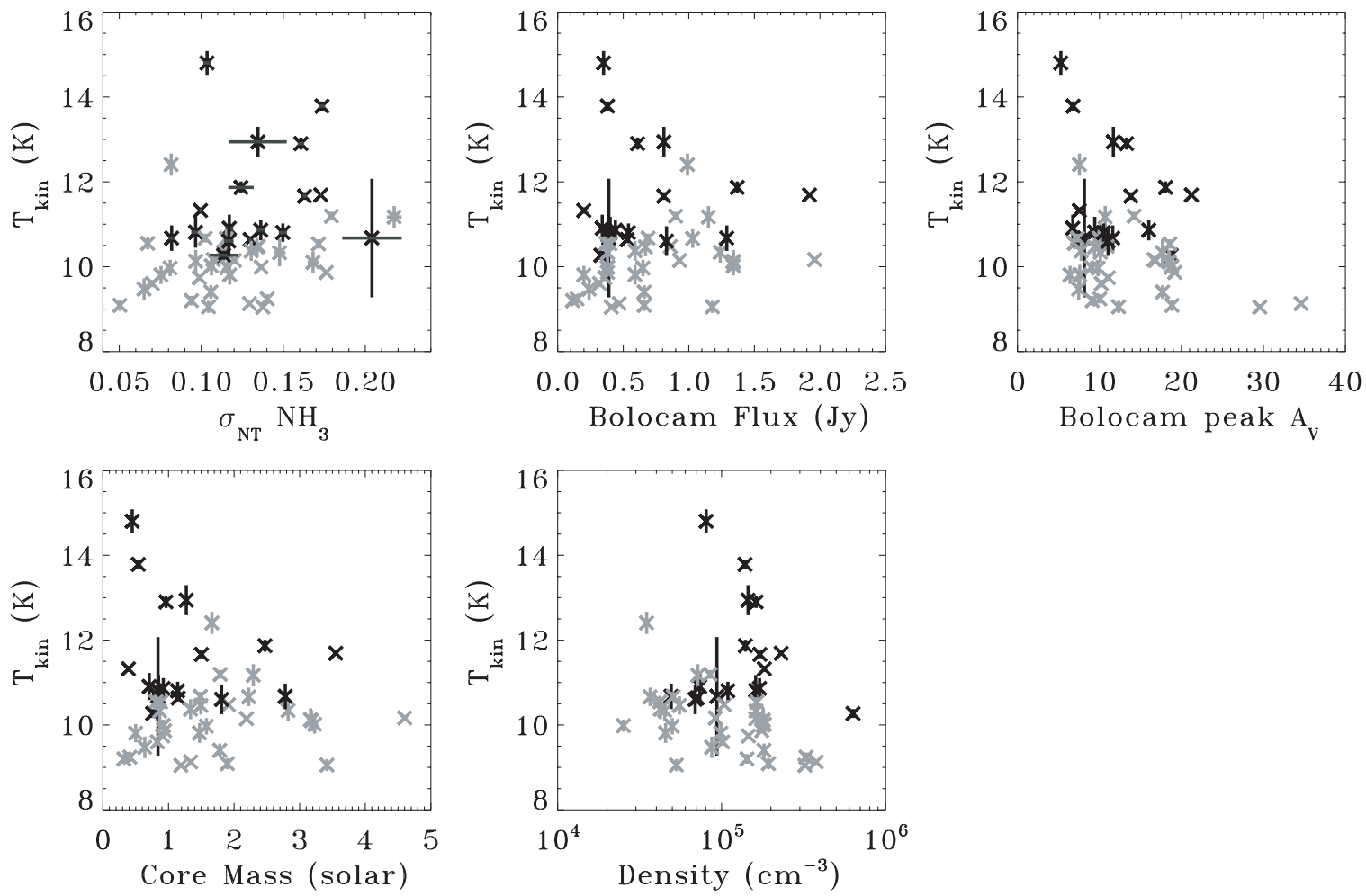

Figure 3. $T_{\text {kin }}$ plotted against properties of the starless cores: (top left) nonthermal line width (one-dimensional velocity dispersion) of $\mathrm{NH}_{3}$; (top middle) Bolocam flux; (top right) peak $A_{V}$; (bottom left) core mass; and (center) average volume density. Starless cores in NGC1333 and IC348 are plotted in black, and field starless cores are plotted in gray. The $1 \sigma$ errors in the fit of $T_{\text {kin }}$ and nonthermal line width are plotted for each core.

derived core properties, but this requires mapping the dust emission from each core at several wavelengths, sampling both the peak of the spectral energy distribution (SED) as well as the Rayleigh-Jeans tail to derive the dust temperature, column density, and emissivity spectral index profiles. Existing surveys of Perseus (Enoch et al. 2006; Kirk et al. 2006) have only sampled the Rayleigh-Jeans portion of the SED, so we do not have accurate estimates of the dust temperatures within the starless cores, and we are forced to use the gas temperature in our calculations.

Note that some of the Bolocam cores, whose numbers are given in Table 1 (Per-Bolo 24, 75, 100, and 122), have been observed in multiple GBT pointings with each position corresponding to different subcondensations within the core. Each of these pointings is assigned the same $1.1 \mathrm{~mm}$ total and peak flux and dimensions as that of the entire Bolocam core, and the peak $A_{V}$, mass, and density are all derived from the overall core Bolocam flux measurements and the $T_{\text {kin }}$ derived for the subcore. An alternative analysis would have been to break up each Bolocam core with more than one $\mathrm{NH}_{3}$ pointing into subcores and assign different fractions of the total $1.1 \mathrm{~mm}$ flux to each portion, but the procedure to do the division would be somewhat arbitrary, just as the decision of which cores should be observed only once and which in multiple positions was not based on an absolute prescription. Since neither analysis is obviously the correct one, we decided to go with the simpler of the two and double count the $1.1 \mathrm{~mm}$ properties of the four Bolocam cores with more than one $\mathrm{NH}_{3}$ observation.

In Figure 3 and Table 3 , we compare $T_{\text {kin }}$ with the $1.1 \mathrm{~mm}$ flux and properties derived from the Enoch et al. (2006) Bolocam map of Perseus. We find no correlation between $T_{\text {kin }}$ and the $1.1 \mathrm{~mm}$ flux, core mass, average volume density, or peak $A_{V}$ for those cores in IC348 and NGC1333. On the other hand, for starless cores in the field there are significant negative correlations between $T_{\text {kin }}$ and the peak $A_{V}$ and average volume density, and a positive correlation between $T_{\text {kin }}$ and the Bolocam flux. If we assume that starless cores are effectively self-shielded from the ISRF because they are not porous, then we would expect higher peak column densities and volume densities to correspond to higher volumetric shielding and lower internal temperatures. It is not obvious why the self-shielding argument does not hold for those starless cores in NGC1333 and IC348. The connection between $1.1 \mathrm{~mm}$ flux and $T_{\text {kin }}$ may be simply that for a given mass, a warmer core will produce more emission, but this argument does not explain why the positive correlation is not seen in cluster starless cores.

\section{CONCLUSIONS}

In this paper, we investigate the temperature distribution of starless cores in Perseus. Although, the range of temperatures derived from $\mathrm{NH}_{3}(1,1)$ and $(2,2)$ pointing is quite narrow, from $\sim 9-15 \mathrm{~K}$, there are several interesting correlations and lack of correlations.

The location of a starless core does affect its temperature. We find that those cores inside the two main star-forming regions in Perseus (IC348 and NGC1333) are warmer than those not in clusters. Although there is no correlation between $T_{\text {kin }}$ and the distance to the nearest Class $0 / \mathrm{I}$ protostar for those starless cores in clusters, starless cores in the field are colder the further away they are from protostars.

Certain properties of the molecular cloud are related to the temperatures of the starless cores embedded in it. $T_{\text {kin }}$ is higher when the extinction-derived column density of the molecular cloud is larger, despite the additional shielding from the ISRF that the higher column density implies, which may be due to the increased pressure on the cores from the molecular cloud in 
higher column density regions. This correlation is not seen when column density along the line of sight in Perseus is measured from FIR dust emission. After controlling for the cluster/field temperature difference, we find that $T_{\text {kin }}$ does not depend on the temperature of the molecular cloud, as measured by $\mathrm{CO}$ or dust emission. The clusters have higher values of $T_{\mathrm{CO}}$ and $T_{\mathrm{d}}$ on average, so presumably the same processes that are heating the clusters are also heating the cores within them. Those starless cores outside NGC1333 and IC348 are warmer in regions with higher ${ }^{13} \mathrm{CO}$ line width, suggesting that the turbulence causing the high line widths makes the cloud more porous to the ISRF, which in turn heats the embedded cores. There is no significant correlation between $\sigma_{\mathrm{CO}}$ and $T_{\text {kin }}$ for those cores in the clusters.

Some internal properties of starless cores are correlated with their temperature. For those starless cores in the field, total 1.1 mm flux is higher in cores that are warmer, and peak column density and average volume density are higher in cores that are colder. A higher temperature naturally leads to more $1.1 \mathrm{~mm}$ flux for a given core mass, and the correlations between $T_{\text {kin }}$ and peak core column density and volume density can be explained by the amount of shielding from the ISRF that the additional material provides. However, it is surprising that none of these correlations exists for starless cores inside the clusters.

We thank the anonymous referee for comments that have improved this paper. S.S. acknowledges support from the Owens Valley Radio Observatory, which is supported by the National Science Foundation through grant AST 05-40399. E.R. was supported by an NSF Astronomy and Astrophysics Postdoctoral Fellowship (AST-0502605) and a Discovery Grant from NSERC of Canada. J.B.F. was supported by a grant from the NRAO Student Observing Support Program (GSSP06-0015). Support was provided to M.L.E. by NASA through the Spitzer Space Telescope Fellowship Program.

\section{REFERENCES}

Belikov, A. N., Kharchenko, N. V., Piskunov, A. E., Schilbach, E., \& Scholz, R.-D. 2002, A\&A, 387, 117

Bergin, E. A., \& Tafalla, M. 2007, ARA\&A, 45, 339

Bertoldi, F., \& McKee, C. F. 1992, ApJ, 395, 140

Bethell, T. J., Zweibel, E. G., Heitsch, F., \& Mathis, J. S. 2004, ApJ, 610, 801

Černis, K., \& Straižys, V. 2003, Balt. Astron., 12, 301
Crapsi, A., Caselli, P., Walmsley, M. C., \& Tafalla, M. 2007, A\&A, 470, 221

Dunham, M. M., Crapsi, A., Evans, N. J., II, Bourke, T. L., Huard, T. L., Myers, P. C., \& Kauffmann, J. 2008, ApJS, 179, 249

Enoch, M. L., Evans, N. J., II, Sargent, A. I., \& Glenn, J. 2008b, ApJ, in press, arXiv:0809.4012

Enoch, M. L., Evans, N. J., II, Sargent, A. I., Glenn, J., Rosolowsky, E., \& Myers, P. 2008a, ApJ, 684, 1240

Enoch, M. L., et al. 2006, ApJ, 638, 293

Evans, N. J., II, Rawlings, J. M. C., Shirley, Y. L., \& Mundy, L. G. 2001, ApJ, 557, 193

Evans, N. J., II, et al. 2003, PASP, 115, 965

Evans, N. J., II, et al. 2007, Final Delivery of Data from the c2d Legacy Project: IRAC and MIPS (Austin, TX: Univ. Texas Dept. Astron.)

Frerking, M. A., Langer, W. D., \& Wilson, R. W. 1982, ApJ, 262, 590

Galli, D., Walmsley, M., \& Gonçalves, J. 2002, A\&A, 394, 275

Goldsmith, P. F. 2001, ApJ, 557, 736

Gonçalves, J., Galli, D., \& Walmsley, M. 2004, A\&A, 415, 617

Goodman, A. A., Barranco, J. A., Wilner, D. J., \& Heyer, M. H. 1998, ApJ, 504, 223

Goodman, A. A., Pineda, J., \& Schnee, S. 2008, ApJ, in press, arXiv:0806.3441

Harvey, P., Merín, B., Huard, T. L., Rebull, L. M., Chapman, N., Evans, N. J., II, \& Myers, P. C. 2007, ApJ, 663, 1149

Hatchell, J., Fuller, G. A., Richer, J. S., Harries, T. J., \& Ladd, E. F. 2007, A\&A, 468, 1009

Jijina, J., Myers, P. C., \& Adams, F. C. 1999, ApJS, 125, 161

Jørgensen, J. K., Johnstone, D., Kirk, H., \& Myers, P. C. 2007, ApJ, 656, 293

Kirk, H., Johnstone, D., \& Di Francesco, J. 2006, ApJ, 646, 1009

Kirk, J. M., Ward-Thompson, D., \& André, P. 2007, MNRAS, 375, 843

Lada, C. J., Muench, A. A., Rathborne, J., Alves, J. F., \& Lombardi, M. 2008, ApJ, 672, 410

Lombardi, M., \& Alves, J. 2001, A\&A, 377, 1023

Miville-Deschênes, M.-A., \& Lagache, G. 2005, ApJS, 157, 302

Ossenkopf, V., \& Henning, T. 1994, A\&A, 291, 943

Pineda, J., Caselli, P., \& Goodman, A. 2008, ApJ, 679, 481

Rebull, L. M., et al. 2007, ApJS, 171, 447

Ridge, N. A., et al. 2006, AJ, 131, 2921

Rosolowsky, E. W., Pineda, J. E., Foster, J. B., Borkin, M. A., Kauffmann, J., Caselli, P., Myers, P. C., \& Goodman, A. A. 2008, ApJS, 175, 509

Schnee, S., Bethell, T., \& Goodman, A. 2006, ApJ, 640, L47

Schnee, S., Li, J., Goodman, A. A., \& Sargent, A. I. 2008, ApJ, 684, 1228

Stamatellos, D., \& Whitworth, A. P. 2003, A\&A, 407, 941

Stamatellos, D., Whitworth, A. P., André, P., \& Ward-Thompson, D. 2004, A\&A, 420,1009

Stamatellos, D., Whitworth, A. P., \& Ward-Thompson, D. 2007, MNRAS, 379, 1390

Swade, D. A. 1989, ApJ, 345, 828

Tafalla, M., Myers, P. C., Caselli, P., Walmsley, C. M., \& Comito, C. 2002, ApJ, 569,815

Walmsley, C. M., \& Ungerechts, H. 1983, A\&A, 122, 164

Zucconi, A., Walmsley, C. M., \& Galli, D. 2001, A\&A, 376, 650 